\title{
Effectiveness of Resuscitation Device when Intubating Suspected COVID-19 Patient at Emergency and Trauma Department
}

\author{
Zainul Ikhwan Ahmad Khusairi \\ Emergency and Trauma Department, Taiping Hospital, Perak DarulRidzuan, Malaysia \\ DOI: http://dx.doi.org/10.31344/ijhhs.v5i0.305
}

Introduction: The rapid spread of COVID-19 had expanded into a pandemic, and thousands of medical staff had been infected worldwide. Thus, the access to personal protective equipment (PPE) for health workers had become a key concern. PPE was essential in providing a physical barrier between microorganism and health care providers. In the case of cardiac arrest, the use of a resuscitation device as PPE had provoked various reactions and complaints from physicians.

Objective: To determine the effectiveness of the use of an intubation box and ergonomic screening booth as a device during intubation procedures on positive COVID-19 patients in the Department of Emergency and Trauma at Taiping Public Hospital.

Methods: The study was designed as a single-blind, prospective, randomized study and was approved by the committee at study location setting. Physicians selected had been given three days of training related to the use of the device as one of the PPE, before being used on patients. The procedure was carried out on three patients with unclear history of COVID-19who required intubation. The time given for each intubation procedure was 5 to 10 seconds with a maximum of three attempts for each patient.

Results: The results found that all three intubation procedures required longer time whereby all procedures took two attempts for each patient. The process of intubation took more than 30 seconds for the first attempt and failed but all cases took about 5 to 10 seconds for second attempts.

Conclusion: This has proven that the use of an intubation box and ergonomic screening booth as a device during intubation procedures was not effective. It is suggested that this device should not be continued by paramedics and a proper management plan is needed to provide appropriate and suitable device innovations.

Keywords: personal protective equipment, resuscitation device, COVID-19, health care workers, emergency department 